\title{
Conformal-field-theoretic analogues of codes and lattices
}

\author{
Yi-Zhi Huang
}

\begin{abstract}
We introduce and study completely-extendable conformal intertwining algebras. Based on results obtained in other papers, various examples are given. Duals of these algebras are constructed and nondegenerate such algebras are defined. We prove that the double dual of such a nondegenrate algebra is equal to itself. We explain using a table that these nondegenerate algebras are the correct conformal-fieldtheoretic analogues of linear binary codes and nondegenerate rational lattices.
\end{abstract}

\section{Introduction}

Codes and lattices play an important role in the study of many mathematical problems and, in particular, they are essential in the study of finite sporadic simple groups (see $[\mathrm{CN}]$ for details). In $\mathbb{B}]$, Borcherds introduced a notion of vertex algebra and announced that the moonshine module constructed by Frenkel, Lepowsky and Meurman in [FLM1] has a natural structure of vertex algebra. In FLM2], Frenkel, Lepowsky and Meurman defined vertex operator algebras, which are vertex algebras equipped with additional data and satisfying additional axioms, and proved that the moonshine module indeed has a structure of a vertex operator algebra (see also [DGM] and [H3] for different proofs). In this major work, Frenkel, Lepowsky and Meurman discovered that vertex operator algebras have many properties analogous to properties of doubly-even codes and even lattices (see [FLM2] for details). They emphasized that linear binary codes, nondegenerate rational lattices and conformal field theories should be viewed as three stages in a hierarchy and the results in one stage should have corresponding results in other stages. 
For the Golay code and Leech lattice, there are uniqueness results which state that any code or lattice satisfying three suitable conditions must be the Golay code or Leech lattice (see [Q] and [CN]). Based on the analogy above, especially the analogy among the Golay code, Leech lattice and moonshine module vertex operator algebra, Frenkel, Lepowsky and Meurman conjectured in ELM2 that the moonshine module should also have an analogous uniqueness property. In [G], Goddard discussed this analogy in more detail and pointed out that one needs to find suitable nonmeromorphic generalizations of vertex (operator) algebras in order to establish a precise correspondence. In particular, one needs a functor on the category of these generalizations corresponding to the dual functors for codes and lattices.

Several nonmeromorphic generalizations of the notion of vertex operator algebra were first given by by Frenkel, Feingold and Ries FFR and Dong and Lepowsky [DL1] [DL2]. The most general one among these notions is the notion of abelian intertwining algebra in DL1 and DL2]. The Verlinde algebras associated to these generalizations are abelian group algebras and involve only one-dimensional representations of the braid groups. A "nonabelian" notion of intertwining operator algebra was introduced in [H5] and studied in [H7] and [H8]. Various examples of intertwining operator algebras were constructed by Lepowsky, Milas and the author in [H3], [H4, [HL4, [HM1] and [HM2] using the results obtained in [HL1], [HL2], [HL3] and [H2].

In the present paper, we introduce a notion of completely-extendable conformal intertwining algebra. The examples of intertwining operator algebras constructed in [H3], [H4], [HL4], [HM1] and [HM2] are examples of completely-extendable conformal intertwining algebras (actually they are "complete"). These examples are constructed using a main theorem in [H2] proving the associativity of intertwining operators. This theorem has a generalization to conformal intertwining algebras. We state this theorem in the present paper but its proof will be given in another paper [H9. We also give some other examples, including the examples constructed from free bosons. Duals of completely-extendable conformal intertwining algebras are constructed and the notion of nondegenerate completely-extendable intertwining operator algebras is introduced in terms of duals. We prove that the double dual of such an algebra is equal to itself. A table filling some blanks in a table given by Goddard in G and containing more items than the one in $G$ ] is given to demonstrate that nondegenerate completely-extendable intertwining operator algebras are the correct conformal-field-theoretic analogues of codes and lattices. We hope that the analogues and correspondence given in 
this paper will lead to at least a strategy to the solution of the uniqueness conjecture of Frenkel, Lepowsky and Meurman for the moonshine module vertex operator algebra. A more conceptual formulation of this correspondence in terms of the language of categories and related results on the correspondence will be given in a future paper.

The present paper is organized as follows: We introduce the notion of locally-grading-restricted conformal intertwining (super)algebra and state the basic properties of these algebras in Section 2. The notion of completelyextendable conformal intertwining (super)algebra is introduced in Section 3. Examples of these algebras are also given in this section. The dual of a completely-extendable conformal intertwining algebra is constructed in Section 4. In the same section, the notion of nondegenerate completelyextendable conformal intertwining algebra is introduced and it is proved that the double dual of such an algebra is equal to itself. In Section 5, we discuss the analogy between codes, lattices and nondegenerate completelyextendable conformal intertwining algebras.

Acknowledgment A large part of the research in the present paper was finished when I visited University of Virginia in the year of 2000. I am grateful to the Department of Mathematics in University of Virginia and Brian Parshall for the hospitality. This research is supported in part by NSF grant DMS-0070800.

\section{Conformal intertwining algebras}

In this section, we relax the grading-restriction conditions for intertwining operator (super)algebras to introduce locally-grading-restricted conformal intertwining (super)algebras. We also give two basic properties of these algebras. Since all these properties are trivial generalizations of the properties of intertwining operator algebras, we omit the proofs here. For details on intertwining operator algebras, see [H5], [H7] and [H8]. We assume that the reader is familiar with the basic notions of vertex operator algebra, module and intertwining operator, as presented in [FLM2] and [FHL].

First we need the following notion of locally-grading-restricted conformal vertex (super)algebra:

Definition 1.1 A conformal vertex algebra of central charge $c$ is a $\mathbb{Z}$-graded vector space equipped with a vertex operator map $Y: V \otimes V \rightarrow V\left[\left[x, x^{-1}\right]\right]$ 
and two distinguished elements 1 (the vacuum) and $\omega$ (the Virasoro element or conformal element) satisfying all the axioms for vertex operator algebras of central charge $c$ except for the two grading-restriction axioms $\operatorname{dim} V_{(n)}<\infty$ for $n \in \mathbb{Z}$ and $V_{(n)}=0$ when the real part of $n$ is sufficiently small. A conformal vertex algebra is said to be locally grading-restricted if (i) for any $m>0, v_{1}, \ldots, v_{m} \in V$, there exists $r \in \mathbb{Z}$ such that the coefficients of the series $Y\left(v_{1}, x_{1}\right) \ldots Y\left(v_{m-1}, x_{m-1}\right) v_{m}$ is in $\coprod_{n>r} V_{(n)}$ and (ii) for any element of the conformal vertex algebra, the module $W=\coprod_{n \in \mathbb{Z}} W_{(n)}$ for the Virasoro algebra generated by this element satisfies the grading-restriction conditions, that is, $\operatorname{dim} W_{(n)}<\infty$ for $n \in \mathbb{Z}$ and $W_{(n)}=0$ when the real part of $n$ is sufficiently small. Modules for a locally-grading-restricted conformal vertex algebra are defined in the same way as modules for a vertex operator algebra except that they are required to be only locally grading-restricted in the sense above. Intertwining operators for a locally-grading-restricted conformal vertex algebra are defined in the obvious way. All the concepts for vertex operator algebras, for example, simple vertex operator algebras and fusion rules, can be generalized to locally-grading-restricted conformal vertex algebras without any difficulty.

Conformal vertex superalgebras are defined similarly except that the underlying vector space has an additional $\mathbb{Z}_{2}$ grading in the commutativity, the Jacobi identity or the skew-symmetry: When both elements are odd, there is an extra minus sign in the term in which the order of the two elements is changed. All the other notions for conformal vertex superalgebras are defined in the obvious way.

Remark 1.2 Condition (i) in the definition above, was first stated in DL2 for abelian intertwining algebras. It guarantees that the convergence and rationality of the matrix elements of products and iterates of vertex operators. Condition (ii) makes sure that all the results involving the Virasoro operators (for example, the geometry of vertex operator algebras in [H1] and [H6]) still hold for these algebras.

We now define locally-grading-restricted conformal intertwining (super)algebra.

Definition 1.3 A locally-grading-restricted conformal intertwining algebra of central charge $c \in \mathbb{C}$ consists of the following data: 
1. A vector space

$$
W=\coprod_{a \in \mathcal{A}} W^{a}
$$

graded by a set $\mathcal{A}$ containing a special element $e$.

2. A structure of locally-grading-restricted conformal vertex algebra of central charge $c$ on $W^{e}$, and a $W^{e}$-module structure on $W^{a}$ for each $a \in \mathcal{A}$.

3. A subspace $\mathcal{V}_{a_{1} a_{2}}^{a_{3}}$ of the space of all intertwining operators of type $\left(\begin{array}{c}W^{a_{3}} \\ W^{a_{1}} W^{a_{2}}\end{array}\right)$ for each triple $a_{1}, a_{2}, a_{3} \in \mathcal{A}$.

These data satisfy the following axioms:

1. The $W^{e}$-module structure on $W^{e}$ is the adjoint module structure. For any $a \in \mathcal{A}$, the space $\mathcal{V}_{e a}^{a}$ is the one-dimensional vector space spanned by the vertex operators defining the $W^{e}$-module structure on $W^{a}$. For any $a_{1}, a_{2} \in \mathcal{A}$ such that $a_{1} \neq a_{2}, \mathcal{V}_{e a_{1}}^{a_{2}}=0$.

2. Convergence properties: For any $m \in \mathbb{Z}_{+}, a_{i}, b_{0}, b_{i}, \in \mathcal{A}, w_{\left(a_{i}\right)} \in W^{a_{i}}$, $\mathcal{Y}_{i} \in \mathcal{V}_{a_{i} b_{i}}^{b_{i-1}}, i=1, \ldots, m, w_{\left(b_{0}\right)}^{\prime} \in\left(W^{b_{0}}\right)^{\prime}$ and $w_{\left(b_{m}\right)} \in W^{b_{m}}$, the series

$$
\left.\left\langle w_{\left(b_{0}\right)}^{\prime}, \mathcal{Y}_{1}\left(w_{\left(a_{1}\right)}, x_{1}\right) \cdots \mathcal{Y}_{m}\left(w_{\left(a_{m}\right)}, x_{m}\right) w_{\left(b_{m}\right)}\right\rangle_{W^{\mu_{1}}}\right|_{x_{i}^{n}=e^{n \log z_{i}, i=1, \ldots, m, n \in \mathbb{C}}}
$$

is absolutely convergent when $\left|z_{1}\right|>\cdots>\left|z_{m}\right|>0$, and consequently (using also other axioms) for any $\mathcal{Y}_{1} \in \mathcal{V}_{a_{1} a_{2}}^{a_{5}}$ and $\mathcal{Y}_{2} \in \mathcal{V}_{a_{5} a_{3}}^{a_{4}}$, the series

$$
\left.\left\langle w_{\left(a_{4}\right)}^{\prime}, \mathcal{Y}_{2}\left(\mathcal{Y}_{1}\left(w_{\left(a_{1}\right)}, x_{0}\right) w_{\left(a_{2}\right)}, x_{2}\right) w_{\left(a_{3}\right)}\right\rangle_{W^{a_{4}}}\right|_{x_{0}^{n}=e^{n \log \left(z_{1}-z_{2}\right)}, x_{2}^{n}=e^{n \log z_{2}, n \in \mathbb{C}}}
$$

is absolutely convergent when $\left|z_{2}\right|>\left|z_{1}-z_{2}\right|>0$.

3. Associativity: For any $a_{1}, a_{2}, a_{3}, a_{4}, a_{5} \in \mathcal{A}$, any $\mathcal{Y}_{1} \in \mathcal{V}_{a_{1} a_{5}}^{a_{4}}$ and $\mathcal{Y}_{2} \in$ $\mathcal{V}_{a_{2} a_{3}}^{a_{5}}$, there exist $\mathcal{Y}_{3}^{a, i} \in \mathcal{V}_{a_{1} a_{2}}^{a}$ and $\mathcal{Y}_{4}^{a, i} \in \mathcal{V}_{a a_{3}}^{a_{4}}$ for $a \in \mathcal{A}$ and $i=1, \ldots, k$ such that only finitely many of $\mathcal{Y}_{3}^{a, i}$ and $\mathcal{Y}_{4}^{a, i}, a \in \mathcal{A}, i=1, \ldots, k$, are nonzero, and for any $w_{\left(a_{i}\right)} \in W^{a_{i}}, i=1,2,3$, and $w_{\left(a_{4}\right)}^{\prime} \in W_{a_{4}}^{\prime}$, the (multivalued) analytic function

$$
\left.\left\langle w_{\left(a_{4}\right)}^{\prime}, \mathcal{Y}_{1}\left(w_{\left(a_{1}\right)}, x_{1}\right) \mathcal{Y}_{2}\left(w_{\left(a_{2}\right)}, x_{2}\right) w_{\left(a_{3}\right)}\right\rangle_{W}\right|_{x_{1}=z_{1}, x_{2}=z_{2}}
$$


defined in the region $\left|z_{1}\right|>\left|z_{2}\right|>0$ and the (multivalued) analytic function

$$
\left.\sum_{a \in \mathcal{A}} \sum_{i=1}^{k}\left\langle w_{\left(a_{4}\right)}^{\prime}, \mathcal{Y}_{4}^{a, i}\left(\mathcal{Y}_{3}^{a, i}\left(w_{\left(a_{1}\right)}, x_{0}\right) w_{\left(a_{2}\right)}, x_{2}\right) w_{\left(a_{3}\right)}\right\rangle_{W^{a_{4}}}\right|_{x_{0}=z_{1}-z_{2}, x_{2}=z_{2}}
$$

defined in the region $\left|z_{2}\right|>\left|z_{1}-z_{2}\right|>0$ are equal in the intersection $\left|z_{1}\right|>\left|z_{2}\right|>\left|z_{1}-z_{2}\right|>0$.

4. Skew-symmetry: For any $a_{1}, a_{2}, a_{3} \in \mathcal{A}$, the restriction of $\Omega_{-1}$ to $\mathcal{V}_{a_{1} a_{2}}^{a_{3}}$ is an isomorphism from $\mathcal{V}_{a_{1} a_{2}}^{a_{3}}$ to $\mathcal{V}_{a_{2} a_{1}}^{a_{3}}$. (Here $\Omega_{-1}$ is the linear map from the space of all intertwining operators of type $\left(\begin{array}{c}a_{3} \\ a_{1} a_{2}\end{array}\right)$ to the space of all intertwining operators of type $\left(\begin{array}{c}a_{3} \\ a_{2} a_{1}\end{array}\right)$ defined by formula

$$
\Omega_{-1}(\mathcal{Y})\left(w_{\left(a_{2}\right)}, x\right) w_{\left(a_{1}\right)}=e^{x L(-1)} \mathcal{Y}\left(w_{\left(a_{1}\right)}, e^{-\pi i} x\right) w_{\left(a_{2}\right)} .
$$

for $w_{\left(a_{1}\right)} \in W^{a_{1}}$ and $w_{\left(a_{2}\right)} \in W^{a_{2}}$. Cf. [HL2].)

A locally-grading-restricted conformal intertwining algebra is said to be meromorphic if intertwining operators are all Laurent series and is said to be $\mathbb{Z}$-graded if weights of elements of $W$ are all integers.

An intertwining operator algebra is a locally-grading-restricted conformal intertwining algebra such that $W^{e}$ is a vertex operator algebra, $W^{a}, a \in \mathcal{A}$, are (grading-restricted) modules for the vertex operator algebra $W^{e}$ and $\mathcal{A}$ is finite. Similarly we have meromorphic intertwining operator algebras and $\mathbb{Z}$-graded intertwining operator algebras.

Locally-grading-restricted conformal intertwining superalgebras and intertwining operator superalgebras are defined similarly in the obvious way.

The locally-grading-restricted conformal intertwining (super)algebra or intertwining operator (super)algebra just defined above is denoted by

$$
\left(W, \mathcal{A},\left\{\mathcal{V}_{a_{1} a_{2}}^{a_{3}}\right\}, \mathbf{1}, \omega\right)
$$

or simply $W$. For simplicity, below we shall often use the abbreviation "l.g.r." for the word "locally-grading-restricted." Clearly, $\mathbb{Z}$-graded l.g.r. conformal intertwining algebras are meromorphic.

Remark 1.4 Actually, l.g.r. conformal intertwining superalgebras can be viewed as conformal intertwining algebras. We shall not discuss this in detail in this paper. See [H9]. See also Remark 2.2 below. 
Now we give two basic properties of l.g.r. conformal intertwining algebras. The proofs of these properties are completely the same as the proofs of the same properties for intertwining operator algebras and will be omitted. The similar properties with possible extra signs for l.g.r. conformal intertwining superalgebras also hold. But we omit the discussions of them in this paper.

The following commutativity generalizes the commutativity for intertwining operator algebras proved in [H4]:

Proposition 1.5 Let $\left(W, \mathcal{A},\left\{\mathcal{V}_{a_{1} a_{2}}^{a_{3}}\right\}, \mathbf{1}, \omega\right)$ be a l.g.r. conformal intertwining algebra. Then we have the following commutativity: For any $a_{1}, \ldots, a_{5} \in \mathcal{A}$, any $\mathcal{Y}_{1} \in \mathcal{V}_{a_{1} a_{5}}^{a_{4}}$ and $\mathcal{Y}_{2} \in \mathcal{V}_{a_{2} a_{3}}^{a_{5}}$, there exist $\mathcal{Y}_{5}^{a, i} \in \mathcal{V}_{a_{2} a}^{a_{4}}$ and $\mathcal{Y}_{6}^{a, i} \in \mathcal{V}_{a_{2} a_{3}}^{a}$ for $a \in \mathcal{A}$ and $i=1, \ldots, k$ such that that only finitely many of $\mathcal{Y}_{5}^{a, i}$ and $\mathcal{Y}_{6}^{a, i}$, $a \in \mathcal{A}, i=1, \ldots, k$, are nonzero, and for any $w_{\left(a_{i}\right)} \in W^{a_{i}}, i=1,2,3$, and $w_{\left(a_{4}\right)}^{\prime} \in W_{a_{4}}^{\prime}$, the (multivalued) analytic function (1.3) defined in the region $\left|z_{1}\right|>\left|z_{2}\right|>0$ and the (multivalued) analytic function

$$
\left.\sum_{a \in \mathcal{A}} \sum_{i=1}^{k}\left\langle w_{\left(a_{4}\right)}^{\prime}, \mathcal{Y}_{5}^{a, i}\left(w_{\left(a_{2}\right)}, x_{2}\right) \mathcal{Y}_{6}^{a, i}\left(w_{\left(a_{1}\right)}, x_{1}\right) w_{\left(a_{3}\right)}\right\rangle_{W^{a_{4}}}\right|_{x_{1}=z_{1}, x_{2}=z_{2}}
$$

defined in the region $\left|z_{2}\right|>\left|z_{1}\right|>0$ are analytic extensions of each other. In the definition of l.g.r. conformal intertwining algebra, skew-symmetry can be replaced by commutativity.

In [H8], generalized rationality for intertwining operator algebras is also proved. In this paper, we do not need the full generalized rationality. What we need is a generalization below of Lemma 4.1 in $\mathrm{H} 8$ to l.g.r. conformal intertwining algebras. Its proof is completely the same as the proof of that lemma in $\mathrm{H} 8$ :

Proposition 1.6 Let $\left(W, \mathcal{A},\left\{\mathcal{V}_{a_{1} a_{2}}^{a_{3}}\right\}, \mathbf{1}, \omega\right)$ be a l.g.r. conformal intertwining algebra. For any $a_{1}, a_{2}, a_{3}, a_{4}, a_{5} \in \mathcal{A}$, any $\mathcal{Y}_{1} \in \mathcal{V}_{a_{1} a_{5}}^{a_{4}}$ and $\mathcal{Y}_{2} \in \mathcal{V}_{a_{2} a_{3}}^{a_{5}}$, there exist a multivalued analytic function defined on $M^{2}=\left\{\left(z_{1}, z_{2}\right) \in \mathbb{C}^{2} \mid z_{1}, z_{2} \neq\right.$ $\left.0, z_{1} \neq z_{2}\right\}, \mathcal{Y}_{3}^{a, i} \in \mathcal{V}_{a_{1} a_{2}}^{a}, \mathcal{Y}_{4}^{a, i} \in \mathcal{V}_{a a_{3}}^{a_{4}}, \mathcal{Y}_{5}^{a, i} \in \mathcal{V}_{a_{2} a}^{a_{4}}$ and $\mathcal{Y}_{6}^{a, i} \in \mathcal{V}_{a_{2} a_{3}}^{a}$, for $a \in \mathcal{A}$ and $i=1, \ldots, k$, such that (1.3), (1.4) and (1.5) are restrictions of this function to their domains $\left|z_{1}\right|>\left|z_{2}\right|>0,\left|z_{2}\right|>\left|z_{1}-z_{2}\right|>0$ and $\left|z_{2}\right|>\left|z_{1}\right|>0$, respectively. 


\section{Complete and completely-extendable con- formal intertwining algebras}

In this section, we first introduce complete and completely-extendable conformal intertwining (super)algebras. In particular, we have complete and completely-extendable intertwining operator (super)algebras. Then we give a construction of complete conformal intertwining (super)algebras generalizing the construction of intertwining operator (super)algebras given in [H2], [H5] and [H\&]. (The details of this generalization will be given in another paper [H9].) We then give examples.

Definition 2.1 A complete conformal intertwining (super)algebra is a l.g.r. conformal intertwining (super)algebra

$$
\left(W, \mathcal{A},\left\{\mathcal{V}_{a_{1} a_{2}}^{a_{3}}\right\}, \mathbf{1}, \omega\right)
$$

satisfying the following conditions:

1. Every irreducible $W^{e}$-module is completely reducible.

2. The set $\left\{W^{a}\right\}_{a \in \mathcal{A}}$ is a complete set of representatives of equivalence classes of irreducible $W^{e}$-modules.

3. For any $a_{1}, a_{2}, a_{3} \in \mathcal{A}, \mathcal{V}_{a_{1} a_{2}}^{a_{3}}$ is the spaces of all intertwining operators of type $\left(\begin{array}{c}W^{a_{3}} \\ W^{a_{1}} W^{a_{2}}\end{array}\right)$.

A completely-extendable conformal intertwining (super)algebra is a l.g.r. conformal intertwining (super)algebra

$$
\left(W, \mathcal{A},\left\{\mathcal{V}_{a_{1} a_{2}}^{a_{3}}\right\}, \mathbf{1}, \omega\right)
$$

satisfying the following conditions:

1. Every irreducible $W^{e}$-module is completely reducible.

2. The $W^{e}$-modules $W^{a}, a \in \mathcal{A}$, are irreducible.

3. For a complete set $\left\{W^{a^{\#}}\right\}_{a \# \in \mathcal{A}^{\#}}$ of representatives of equivalence classes of irreducible $W^{e}$-modules, the direct sum $W^{\#}=\coprod_{a^{\#} \in \mathcal{A}^{\#}} W^{a^{\#}}$ equipped with the index set $\mathcal{A}^{\#}$, the vacuum and the Virasoro element of $W^{e}$ 
and the spaces $\left(\mathcal{V}^{\#}\right)_{a_{1}^{\#} a_{2}^{\#}}^{a_{3}^{\#}}, a_{1}^{\#}, a_{2}^{\#}, a_{3}^{\#} \in \mathcal{A}^{\#}$, of all intertwining operators of type $\left(\begin{array}{c}a_{3}^{\#} \\ a_{1}^{\#} a_{2}^{\#}\end{array}\right)$, is also a l.g.r. conformal intertwining (super)algebra (which by definition is complete).

By definition, different l.g.r. conformal intertwining (super)algebras obtained from different sets of representatives of equivalence classes of irreducible $W^{e}$-modules are naturally isomorphic. We call $W^{\#}$ a complete extension of $W$.

Complete conformal vertex (super)algebras, completely-extendable conformal vertex (super)algebras, complete vertex operator (super)algebras, completelyextendable vertex operator (super)algebras, meromorphic complete intertwining algebras and $\mathbb{Z}$-graded complete intertwining algebras are defined in the obvious way.

From the definition, we see that a completely-extendable conformal intertwining (super)algebra is a complete extension of itself if and only if it is complete.

Remark 2.2 As remarked in Remark 1.4, a complete conformal intertwining superalgebra can be viewed as a conformal intertwining algebra. But it is not complete when viewed as a conformal intertwining algebra.

In [H2], for a rational vertex operator algebra satisfying suitable conditions, the associativity of intertwining operators was proved. Since the associativity is the only nontrivial property one needs to verify when one tries to construct an intertwining operator algebras from representations of a rational vertex operator algebra, this result in [H2] in fact gives a construction of intertwining operator algebras. By construction, these intertwining operator algebras are all complete. The same construction, except for the change of some signs, works for rational vertex operator superalgebras satisfying suitable conditions (see the relevant discussions in HM1]).

In the case of familiar vertex operator (super)algebras (certain vertex operator (super)algebras associated to the Virasoro algebra, affine Lie algebras, the $N=1$ and $N=2$ Neveu-Schwarz algebras and related algebras), the conditions needed were verified in [H4], [HL4], [HM1] and [HM2] and thus we obtain complete intertwining operator (super)algebras from representations of these vertex operator (super)algebras. 
This construction of complete intertwining operator (super)algebras has a generalization to a construction of complete conformal intertwining (super)algebras. Here we state the theorem but its proof will be given in a long paper [H9] in preparation on intertwining algebras.

We shall formulate the results only for conformal intertwining algebras. The super case is the same except for the change of some signs. To formulate the result precisely, we need the following notion which in the case of vertex operator algebra was first introduced in [H2]:

Definition 2.3 Let $V$ be a l.g.r. conformal vertex algebra. We say that products of intertwining operators for $V$ satisfy the convergence and extension property if for any intertwining operators $\mathcal{Y}_{1}$ and $\mathcal{Y}_{2}$ of types $\left(\begin{array}{c}W_{0} \\ W_{1} W_{4}\end{array}\right)$ and $\left(\begin{array}{c}W_{4} \\ W_{2} W_{3}\end{array}\right)$, respectively, there exists an integer $N$ (depending only on $\mathcal{Y}_{1}$ and $\left.\mathcal{Y}_{2}\right)$, and for any $w_{(1)} \in W_{1}, w_{(2)} \in W_{2}, w_{(3)} \in W_{3}, w_{(0)}^{\prime} \in W_{0}^{\prime}$, there exist $j \in \mathbb{N}, r_{i}, s_{i} \in \mathbb{R}, i=1, \ldots, j$, and analytic functions $f_{i}(z)$ on $|z|<1$, $i=1, \ldots, j$, such that

$$
\left.\left\langle w_{(0)}^{\prime}, \mathcal{Y}_{1}\left(w_{(1)}, x_{1}\right) \mathcal{Y}_{2}\left(w_{(2)}, x_{2}\right) w_{(3)}\right\rangle\right|_{x_{1}=z_{1}, x_{2}=z_{2}}
$$

is absolutely convergent when $\left|z_{1}\right|>\left|z_{2}\right|>0$ and can be analytically extended to the multivalued analytic function

$$
\sum_{i=1}^{j} z_{2}^{r_{i}}\left(z_{1}-z_{2}\right)^{s_{i}} f_{i}\left(\frac{z_{1}-z_{2}}{z_{2}}\right)
$$

when $\left|z_{2}\right|>\left|z_{1}-z_{2}\right|>0$. In the case that $V$ is a vertex operator algebra, we require in addition that

$$
\text { wt } w_{(1)}+\text { wt } w_{(2)}+s_{i}>N, \quad i=1, \ldots, j \text {. }
$$

Remark 2.4 It is easy to see that if the associativity of intertwining operators holds (see the associativity axiom in Definition 1.3), then products of intertwining operators do satisfy the convergence and extension property. So this condition is in fact necessary for the associativity of intertwining operators. 
Theorem 2.5 Let $V$ be a l.g.r. conformal vertex algebra and $\left\{W^{a}\right\}_{a \in \mathcal{A}}$ a complete set of representatives of equivalence classes of irreducible $V$ modules. Assume that every $V$-module is completely reducible, every irreducible $V$-module is $\mathbb{R}$-graded, products of intertwining operators for $V$ satisfy the convergence and extension property, and for any $a_{1}, a_{2} \in \mathcal{A}$, there are only finitely many $a_{3} \in \mathcal{A}$ such that there exist nonzero intertwining operators of type $\left(\begin{array}{c}a_{3} \\ a_{1} a_{2}\end{array}\right)$. Then the $\mathbb{R}$-graded vector space $\coprod_{a \in \mathcal{A}} W^{a}$ together with the index set $\mathcal{A}$ with the special element e such that $W^{e}$ is isomorphic to a subalgebra of $V$, the spaces $\mathcal{V}_{a_{1} a_{2}}^{a_{3}}, a_{1}, a_{2}, a_{3} \in \mathcal{A}$, of all intertwining operators of type $\left(\begin{array}{c}a_{3} \\ a_{1} a_{2}\end{array}\right)$, the vacuum $\mathbf{1} \in W^{e}$ and the Virasoro element $\omega \in W^{e}$ is a conformal intertwining algebra. If $V$ is in addition simple in the sense that $V$ as a $V$-module is irreducible, this conformal intertwining algebra is complete.

As is mentioned above, the proof of this theorem will be given in [H9].

The construction of intertwining operator algebras obtained in [H2] and [H4 can be obtained as an easy consequence of the result above. Here we state one theorem obtained in [H2 and [H4] and formulated in terms of intertwining operator algebras first in [H5]. We need several generalizations of the notion of module for a vertex operator algebra. A generalized $V$-module is a $\mathbb{C}$-graded vector space equipped with a vertex operator map satisfying all the axioms for a $V$-module except for the two grading-restriction conditions. A generalized $V$-module is said to be locally-grading-restricted or simply l.g.r. if it is a $V$-module when $V$ is viewed as a l.g.r. conformal vertex algebra. A l.g.r. generalized $V$-module is said to be lower-truncated if there exists $N \in \mathbb{Z}$ such that its homogeneous subspace of weight $n$ is 0 when the real part of $n$ is less than $N$.

Corollary 2.6 Let $V$ be a rational vertex operator algebra and $\left\{W^{i}\right\}_{i=1}^{m} a$ set of representatives of equivalence classes of irreducible $V$-modules. Assume that every irreducible $V$-module is $\mathbb{R}$-graded, products of intertwining operators for $V$ satisfy the convergence and extension property (including the additional condition (2.1)), and every finitely-generated lower-truncated l.g.r. generalized $V$-module is a $V$-module. Then the $\mathbb{R}$-graded vector space $\coprod_{i=1}^{m} W^{i}$ together with the finite set $\mathcal{A}=\{1, \ldots, m\}$ with the special element e such that $W^{e}$ is isomorphic to a subalgebra of $V$, the spaces $\mathcal{V}_{a_{1} a_{2}}^{a_{3}}$, $a_{1}, a_{2}, a_{3} \in \mathcal{A}$, of all intertwining operators of type $\left(\begin{array}{c}a_{3} \\ a_{1} a_{2}\end{array}\right)$, the vacuum $\mathbf{1} \in V$ and the Virasoro element $\omega \in V$ is a intertwining operator algebra. If $V$ 
is in addition simple in the sense that $V$ is irreducible as a $V$-module, then $\coprod_{i=1}^{m} W^{i}$ is complete.

The proof of this corollary using Theorem 2.5 will also be given in [H9].

Now we give examples of complete and complete-extendable conformal intertwining operator algebras. Since these examples are mostly highly nontrivial, we refer the reader to the references for details except for algebras given in Examples 2.7 and 2.9.

Example 2.7 Rational vertex operator algebras with themselves as the only irreducible modules up to isomorphisms: Any such algebra is a complete vertex operator algebra. In particular, the moonshine module $V^{\natural}$ is a complete vertex operator algebra.

Example 2.8 Lattice abelian intertwining algebras: In [DL2], Dong and Lepowsky constructed abelian intertwining algebras associated to nondegenerate lattices. These abelian intertwining algebras are completely-extendable conformal intertwining algebras.

Example 2.9 Free bosons: Let $\mathfrak{h}_{\mathbb{R}}$ be an $m$-dimensional vector space over $\mathbb{R}$ equipped with a nondegenerate bilinear form $(\cdot, \cdot)$. We choose a basis $\left\{h_{i}\right\}_{i=1}^{m}$ of $\mathfrak{h}_{\mathbb{R}}$ which is orthonormal in the sense that $\left(h_{i}, h_{j}\right)=\eta_{i j}$ where $\eta_{i j}=0$ when $i \neq j$ and $\eta_{i i}= \pm 1$. Let $\mathfrak{h}=\mathfrak{h}_{\mathbb{R}} \otimes_{\mathbb{R}} \mathbb{C}$ be the complexification of $\mathfrak{h}_{\mathbb{R}}$ with the bilinear form obtained by extending linearly from the one on $\mathfrak{h}_{\mathbb{R}}$. For convenience, we use the same notations $h_{i}$ to denote $h_{i} \otimes 1 \in \mathfrak{h}$ for $i=1, \ldots, m$. We view $\mathfrak{h}$ as an abelian Lie algebra and consider the $\mathbb{Z}$-graded untwisted affine Lie algebra $\tilde{\mathfrak{h}}=\coprod_{n \in \mathbb{Z}} \mathfrak{h} \otimes t^{n} \oplus \mathbb{C} k \oplus \mathbb{C} d$, its Heisenberg subalgebra $\hat{\mathfrak{h}}_{\mathbb{Z}}=\coprod_{n \in \mathbb{Z}, n \neq 0} \mathfrak{h} \otimes t^{n} \oplus \mathbb{C} k$ and the subalgebra $\hat{\mathfrak{h}}_{\mathbb{Z}}^{-}=\coprod_{n<0} \mathfrak{h} \otimes t^{n}$. The symmetric algebra $S\left(\hat{\mathfrak{h}}_{\mathbb{Z}}^{-}\right)$over $\hat{\mathfrak{h}}_{\mathbb{Z}}^{-}$is a $\mathbb{Z}$-graded $\hat{\mathfrak{h}}_{\mathbb{Z}}$-irreducible $\tilde{\mathfrak{h}}$-module. There is a unique vertex operator algebra structure on $S\left(\hat{\mathfrak{h}}_{\mathbb{Z}}^{-}\right)$such that 1 is the vacuum, $\omega=\frac{1}{2} \sum_{i=1}^{m} \eta_{i i} h_{i}(-1)^{2}$ is the Virasoro element and for any $h \in \mathfrak{h}, Y(h(-1), x)=\sum_{n \in \mathbb{Z}} h(n) x^{-n-1}$.

Given any $g \in \mathfrak{h}_{\mathbb{R}}, S\left(\hat{\mathfrak{h}}_{\mathbb{Z}}^{-}\right) \otimes \mathbb{C} g$ is a $\hat{\mathfrak{h}}_{\mathbb{Z}}$-irreducible $\tilde{\mathfrak{h}}$-module such that for any $h \in \mathfrak{h}, h(0)(1 \otimes g)=(h, g)(1 \otimes g)$. This $\tilde{\mathfrak{h}}$-module has a unique irreducible module structure for the vertex operator algebra $S\left(\hat{\mathfrak{h}}_{\mathbb{Z}}^{-}\right)$such that for any $h \in \mathfrak{h}, Y(h(-1), x)=\sum_{n \in \mathbb{Z}} h(n) x^{-n-1}$. It is easy to show that any irreducible 
module for $S\left(\hat{\mathfrak{h}}_{\mathbb{Z}}^{-}\right)$is isomorphic to such a module, such modules with different $g \in \mathfrak{h}_{\mathbb{R}}$ are not isomorphic, and any module for $S\left(\hat{\mathfrak{h}}_{\mathbb{Z}}^{-}\right)$is completely reducible. It is easy to show that $\coprod_{g \in \mathfrak{h}_{\mathbb{R}}} S\left(\hat{\mathfrak{h}}_{\mathbb{Z}}^{-}\right) \otimes \mathbb{C} g$ is a l.g.r. conformal intertwining algebra, and by definition, it is complete.

Example 2.10 The $\mathbb{Z}_{2}$-orbifold theory underlying the moonshine module: In [H3], the author constructed an abelian intertwining algebra on the direct sum of the Leech lattice vertex operator algebra and its irreducible twisted module. It is a complete intertwining operator algebra.

Example 2.11 Minimal models: In [H4], the author constructed the intertwining operator algebras associated to the minimal models. They are complete.

Example 2.12 WNZW models: In [HL6], Lepowsky and the author constructed the intertwining operator algebras associated to the WNZW models. They are complete.

Example 2.13 $N=1$ superconformal minimal models: In [HM1], Milas and the author constructed the intertwining operator superalgebras associated to the $N=1$ superconformal minimal models. They are complete.

Example 2.14 $N=2$ superconformal unitary models: In [HM2, Milas and the author constructed the intertwining operator superalgebras associated to the $N=2$ superconformal unitary models. They are complete.

Example 2.15 Other examples: Let $V$ be a rational vertex operator algebra containing a subalgebra isomorphic to a tensor product algebra of the vertex operator algebras associated to the minimal models or the WNZW models or $N=1$ superconformal minimal models or $N=2$ superconformal unitary models. In [H4] or [HL6] or [HM1] or [HM2], respectively, it was proved that the direct sum of all irreducible $V$-modules in a complete set of equivalence classes of all irreducible modules has a structure of intertwining operator algebra. It is complete. 


\section{Duals of completely-extendable intertwin- ing operator algebras}

In this section, we construct duals of completely-extendable conformal intertwining algebras and prove their basic properties. We also introduce nondegenerate completely-extendable conformal intertwining algebras and prove that for these algebras, their double duals are equal to themselves.

Let

$$
\left(W, \mathcal{A},\left\{\mathcal{V}_{a_{1} a_{2}}^{a_{3}}\right\}, \mathbf{1}, \omega\right)
$$

be a completely-extendable conformal intertwining algebra. In this and next section, we fix a complete extension

$$
\left(W^{\#}, \mathcal{A}^{\#},\left\{\left(\mathcal{V}^{\#}\right)_{a_{1}^{\#} a_{2}^{\#}}^{a_{3}^{\#}}\right\}, \mathbf{1}, \omega\right)
$$

$W$. Consider $a^{\#} \in \mathcal{A}^{\#}$ such that for any $a \in \mathcal{A}, a_{1}^{\#} \in \mathcal{A}^{\#}$ and any $\mathcal{Y} \in \mathcal{V}_{a a}^{a_{1}^{\#}}$, the image of $\mathcal{Y}: W^{a} \otimes W^{a^{\#}} \mapsto W^{a_{1}^{\#}}\{x\}$ is in $W^{a_{1}^{\#}}\left[\left[x^{-1}, x\right]\right]$ (and thus in $\left.W^{a_{1}^{\#}}((x))\right)$. We denote the set of all such elements $a^{\#} \in \mathcal{A}^{\#}$ by $\mathcal{A}^{\circ}$. We shall use $a^{\circ}, a_{1}^{\circ}, \ldots$, to denote elements of $\mathcal{A}^{\circ}$. Let $W^{\circ}=\coprod_{a^{\circ} \in \mathcal{A}^{\circ}} W^{a^{\circ}}$. First we have the following result:

Proposition 3.1 Let $a_{1}^{\circ}, a_{2}^{\circ} \in \mathcal{A}^{\circ}$ and $a_{3}^{\#} \in \mathcal{A}^{\#}$. If $\left(\mathcal{V}^{\#}\right)_{a_{1}^{\#} a_{2}^{\#}}^{a_{3}^{\#}} \neq 0$, then $a_{3}^{\#} \in \mathcal{A}^{\circ}$.

Proof. Suppose that $a_{3}^{\#} \notin \mathcal{A}^{\circ}$. Then by definition, there exist $a \in \mathcal{A}$, $a_{4}^{\#} \in \mathcal{A}^{\#}, w_{(a)} \in W^{a}, w_{\left(a_{1}^{\#}\right)} \in W^{a_{1}^{\#}}, w_{\left(a_{2}^{\#}\right)} \in W^{a_{2}^{\#}}, \mathcal{Y}_{1} \in\left(\mathcal{V}^{\#}\right)_{a a_{3}^{\#}}^{a_{\#}^{\#}}$ and $\mathcal{Y}_{2} \in\left(\mathcal{V}^{\#}\right)_{a_{1}^{\#} a_{2}^{\#}}^{a_{\#}^{\#}}$ such that

$$
\mathcal{Y}_{1}\left(w_{(a)}, x_{1}\right) \mathcal{Y}_{2}\left(w_{\left(a_{1}^{\#}\right)}, x_{2}\right) w_{\left(a_{2}^{\#}\right)}
$$

has terms in nonintegral powers of $x_{1}$. On the other hand, by Proposition 1.6, for any $w_{\left(a_{4}^{\#}\right)}^{\prime} \in\left(W^{a_{4}^{\#}}\right)^{\prime}$,

$$
\left.\left\langle w_{\left(a_{4}^{\#}\right)}^{\prime}, \mathcal{Y}_{1}\left(w_{(a)}, x_{1}\right) \mathcal{Y}_{2}\left(w_{\left(a_{1}^{\#}\right)}, x_{2}\right) w_{\left(a_{2}^{\#}\right)}\right\rangle_{W^{a}}\right|_{x_{1}=z_{1}, x_{2}=z_{2}}
$$


is absolutely convergent in the region $\left|z_{1}\right|>\left|z_{2}\right|>0$ and can be analytically extended to a (multivalued) analytic function $f$ on $M^{2}=\left\{\left(z_{1}, z_{2}\right) \in\right.$ $\left.\mathbb{C}^{2} \mid z_{1}, z_{2} \neq 0, z_{1} \neq z_{2}\right\}$. Since

$$
\mathcal{Y}_{1}\left(w_{(a)}, x_{1}\right) \mathcal{Y}_{2}\left(w_{\left(a_{1}^{\#}\right)}, x_{2}\right) w_{\left(a_{2}^{\#}\right)}
$$

has nonintegral power terms in $x_{1}$, there exists $w_{\left(a_{4}^{\#}\right)}^{\prime} \in\left(W^{a_{4}^{\#}}\right)^{\prime}$ such that $f$ is multivalued in $z_{1}$. But by Proposition 1.6 again, there exist $\mathcal{Y}_{3}^{a_{5}^{\#}, i} \in\left(\mathcal{V}^{\#}\right)_{a a_{1}^{\#}}^{a_{5}^{\#}}$, $\mathcal{Y}_{4}^{a_{5}^{\#}, i} \in\left(\mathcal{V}^{\#}\right)_{a_{5}^{\#} a_{2}^{\#}}^{a_{\#}^{\#}}, \mathcal{Y}_{5}^{a_{5}^{\#}, i} \in\left(\mathcal{V}^{\#}\right)_{a_{2}^{\#} a_{5}^{\#}}^{a_{\#}^{\#}}$ and $\mathcal{Y}_{6}^{a_{5}^{\#}, i} \in\left(\mathcal{V}^{\#}\right)_{a a_{2}^{\#}}^{a_{5}^{\#}}$, for $a_{5}^{\#} \in \mathcal{A}^{\#}$ and $i=1, \ldots, k$, such that $f$ is equal to

$$
\left.\sum_{a_{5}^{\#} \in \mathcal{A}^{\#}} \sum_{i=1}^{k}\left\langle w_{\left(a_{4}^{\#}\right)}^{\prime}, \mathcal{Y}_{4}^{a_{5}^{\#}, i}\left(\mathcal{Y}_{3}^{a_{5}^{\#}, i}\left(w_{(a)}, x_{0}\right) w_{\left(a_{1}^{\#}\right)}, x_{2}\right) w_{\left(a_{2}^{\#}\right)}\right\rangle_{W^{a_{4}^{\#}}}\right|_{x_{0}=z_{1}-z_{2}, x_{2}=z_{2}}
$$

in the region $\left|z_{2}\right|>\left|z_{1}-z_{2}\right|>0$ and is equal to and

$$
\left.\sum_{a_{5}^{\#} \in \mathcal{A}^{\#}} \sum_{i=1}^{k}\left\langle w_{\left(a_{4}^{\#}\right)}^{\prime}, \mathcal{Y}_{5}^{a^{\#}, i}\left(w_{\left(a_{1}^{\#}\right)}, x_{2}\right) \mathcal{Y}_{6}^{a^{\#}, i}\left(w_{(a)}, x_{1}\right) w_{\left(a_{2}^{\#}\right)}\right\rangle_{W^{a_{4}^{*}}}\right|_{x_{1}=z_{1}, x_{2}=z_{2}}
$$

in the region $\left|z_{2}\right|>\left|z_{1}\right|>0$. Since $a_{1}^{\circ}, a_{2}^{\circ} \in \mathcal{A}^{\circ}$. So $\mathcal{Y}_{3}^{a_{5}^{\#}, i}\left(w_{(a)}, x_{0}\right) w_{\left(a_{1}^{\#}\right)}$ and $\mathcal{Y}_{6}^{a^{\#}, i}\left(w_{(a)}, x_{1}\right) w_{\left(a_{2}^{\#}\right)}, a_{5}^{\#} \in \mathcal{A}^{\#}, i=1, \ldots, k$, have only terms in integral power of $x_{0}$ and $x_{1}$, respectively. Thus for fixed $z_{2}, f$ as a function of $z_{1}$ is single-valued near the singularities $z_{1}=z_{2}$ and $z_{1}=0$. But for fixed $z_{2}, f$ as a function of $z_{1}$ has only three singularities, $z_{1}=\infty, z_{1}=z_{2}$ and $z_{1}=0$. It is impossible to have such an analytic function which is single-valued near two singularities but multivalued near the other singularity. Contradiction.

The main result of this section is the following:

Theorem 3.2 The direct sum $W^{\circ}=\coprod_{a^{\circ} \in \mathcal{A}^{\circ}} W^{a^{\circ}}$, equipped with $\mathcal{A}^{\circ}$, the spaces

$$
\left(\mathcal{V}^{\circ}\right)_{a_{1}^{\circ} a_{2}^{\circ}}^{a^{\circ}}=\left(\mathcal{V}^{\#}\right)_{a_{1}^{\circ} a_{2}^{\circ}}^{a_{\circ}^{\circ}}
$$

of intertwining operators, the vacuum and the Virasoro element of $W^{e}$, is an conformal intertwining algebra. In addition, it is completely extendable and its complete extension is equal to the complete extension of $W$. 
Proof. We need only prove the associativity and the skew-symmetry. Let $\mathcal{Y}_{1} \in\left(\mathcal{V}^{\circ}\right)_{a_{1}^{\circ} a_{5}^{\circ}}^{a_{0}^{\circ}}$ and $\mathcal{Y}_{2} \in\left(\mathcal{V}^{\circ}\right)_{a_{2}^{\circ} a_{3}^{\circ}}^{a_{0}^{\circ}}$. By the associativity for the completely extension $W^{\#}$ of $W$, there exist $\mathcal{Y}_{3}^{a^{\#} i} \in\left(\mathcal{V}^{\#}\right)_{a_{1}^{a} a_{2}^{\circ}}^{a_{0}}$ and $\mathcal{Y}_{4}^{a^{\#}, i} \in\left(\mathcal{V}^{\#}\right)_{a_{4}^{\#} a_{3}^{\circ}}^{a_{0}^{\circ}}$ for all $a^{\#} \in \mathcal{A}^{\#}$ and $i=1, \ldots, k$ such that the (multivalued) analytic function

$$
\left.\left\langle w_{\left(a_{4}^{\circ}\right)}^{\prime}, \mathcal{Y}_{1}\left(w_{\left(a_{1}^{\circ}\right)}, x_{1}\right) \mathcal{Y}_{2}\left(w_{\left(a_{2}^{\circ}\right)}, x_{2}\right) w_{\left(a_{3}^{\circ}\right)}\right\rangle_{W^{a_{4}^{4}}}\right|_{x_{1}=z_{1}, x_{2}=z_{2}}
$$

defined in the region $\left|z_{1}\right|>\left|z_{2}\right|>0$ and the (multivalued) analytic function

$$
\left.\sum_{a^{\#} \in \mathcal{A}^{\#}} \sum_{i=1}^{k}\left\langle w_{\left(a_{4}^{\circ}\right)}^{\prime}, \mathcal{Y}_{4}^{a^{\#}, i}\left(\mathcal{Y}_{3}^{a^{\#}, i}\left(w_{\left(a_{1}^{\circ}\right)}, x_{0}\right) w_{\left(a_{2}^{\circ}\right)}, x_{2}\right) w_{\left(a_{3}^{\circ}\right)}\right\rangle_{W^{a_{4}^{\circ}}}\right|_{x_{0}=z_{1}-z_{2}, x_{2}=z_{2}}
$$

defined in the region $\left|z_{2}\right|>\left|z_{1}-z_{2}\right|>0$ are equal in the intersection $\left|z_{1}\right|>$ $\left|z_{2}\right|>\left|z_{1}-z_{2}\right|>0$. By Proposition 3.1, we see that $\mathcal{Y}_{3}^{a^{\#}, i}$ must be 0 if $a^{\#} \notin \mathcal{A}^{\circ}$, proving the associativity.

The skew-symmetry is obvious from the definition.

The completely-extendable conformal intertwining algebra $W^{\circ}$ is called the dual of $W$. If $W^{\circ}=W$, we say that $W$ is self-dual.

Proposition 3.3 Let $W$ be a completely-extendable conformal intertwining algebra. Then $\mathcal{A} \subset\left(\mathcal{A}^{\circ}\right)^{\circ}, W \subset\left(W^{\circ}\right)^{\circ},\left(\left(\mathcal{A}^{\circ}\right)^{\circ}\right)^{\circ}=\mathcal{A}$ and $\left(\left(W^{\circ}\right)^{\circ}\right)^{\circ}=W^{\circ}$.

Proof. Let $a \in \mathcal{A}$. Then by the definition of $W^{\circ}$, for any $a^{\circ} \in \mathcal{A}^{\circ}, a^{\#} \mathcal{A}$ and any $\mathcal{Y} \in \mathcal{V}_{a a^{\circ}}^{a^{\#}}$, the image of $\mathcal{Y}: W^{a} \otimes W^{a^{\circ}} \mapsto W^{a^{\#}}\{x\}$ is in $W^{a_{1}^{\#}}\left[\left[x^{-1}, x\right]\right]$. Equivalently, $\Omega_{-1}(\mathcal{Y}): W^{a^{\circ}} \otimes W^{a} \mapsto W^{a^{\#}}\{x\}$ is in $W^{a_{1}^{\#}}\left[\left[x^{-1}, x\right]\right]$. Since $\Omega_{-1}$ is a linear isomorphism, we conclude that for any $a^{\circ} \in \mathcal{A}^{\circ}, a^{\#} \in \mathcal{A}$ and any $\mathcal{Y} \in \mathcal{V}_{a^{\circ} a}^{a \#}$, the image of $\mathcal{Y}: W^{a^{\circ}} \otimes W^{a} \mapsto W^{a^{\#}}\{x\}$ is in $W^{a^{\#}}\left[\left[x^{-1}, x\right]\right]$. Thus by definition, $a \in\left(\mathcal{A}^{\circ}\right)^{\circ}$. Since $a$ is arbitrary, $\mathcal{A} \subset\left(\mathcal{A}^{\circ}\right)^{\circ}$ and $W \subset\left(W^{\circ}\right)^{\circ}$.

Now we know that $\mathcal{A} \subset\left(\left(\mathcal{A}^{\circ}\right)^{\circ}\right)^{\circ}$ and $W^{\circ} \subset\left(\left(W^{\circ}\right)^{\circ}\right)^{\circ}$. To prove the second conclusion, we need only prove that $\left(\left(\mathcal{A}^{\circ}\right)^{\circ}\right)^{\circ} \subset \mathcal{A}^{\circ}$. Let $a^{\text {oo }} \in$ $\left(\left(\mathcal{A}^{\circ}\right)^{\circ}\right)^{\circ}$. By definition, for any $a^{\circ \circ} \in\left(\mathcal{A}^{\circ}\right)^{\circ}, a^{\#} \in \mathcal{A}$ and any $\mathcal{Y} \in \mathcal{V}_{a^{\circ \circ}}^{a^{\#}} a^{\circ \circ \circ}$, the image of $\mathcal{Y}: W^{a^{\circ \circ}} \otimes W^{a^{\circ \circ 0}} \mapsto W^{a^{\#}}\{x\}$ is in $W^{a^{\#}}\left[\left[x^{-1}, x\right]\right]$. Since $\mathcal{A} \subset\left(\mathcal{A}^{\circ}\right)^{\circ}$, we have, in particular, for any $a \in \mathcal{A}, a^{\#} \in \mathcal{A}$ and any $\mathcal{Y} \in \mathcal{V}_{a a^{\circ \circ}}^{a^{\circ}}$, the image of $\mathcal{Y}: W^{a} \otimes W^{a^{\circ 00}} \mapsto W^{a^{\#}}\{x\}$ is in $W^{a^{\#}}\left[\left[x^{-1}, x\right]\right]$. Thus by definition, $a^{\circ 00} \in \mathcal{A}^{\circ}$, proving $\left(\left(\mathcal{A}^{\circ}\right)^{\circ}\right)^{\circ} \subset \mathcal{A}^{\circ}$.

Example 3.4 Any vertex operator algebra in Example 2.7 is self-dual. 
Example 3.5 Any simple l.g.r. conformal vertex algebra satisfying the conditions in Theorem 2.5 is completely extendable by the theorem. Its dual is its complete extension.

We need the following notion:

Definition 3.6 A completely-extendable conformal intertwining algebra

$$
\left(W, \mathcal{A},\left\{\mathcal{V}_{a_{1} a_{2}}^{a_{3}}\right\}, \mathbf{1}, \omega\right)
$$

is said to be nondegenerate if it is the dual of a completely-extendable conformal intertwining algebra. It is said to be degenerate if it is not nondegenerate.

For simplicity, we shall often call a nondegenerate completely-extendable conformal intertwining algebra simply a nondegenerate conformal intertwining algebra.

We have:

Theorem 3.7 Let $W$ be a completely-extendable conformal intertwining algebra. Then $W$ is nondegenerate if and only if $\left(W^{\circ}\right)^{\circ}=W$.

Proof. The "if" part follows from the definition. The only if part follows from Proposition 3.3 .

The examples of complete-extendable conformal intertwining algebras given in the preceding section are all nondegenerate. Here we give an example of degenerate completely-extendable intertwining operator algebra.

Example 3.8 Let $W^{0}$ be a vertex operator algebra satisfying the conditions in Theorem 2.5 and $W^{1}$ an irreducible $W^{0}$-module. We consider $W=$ $W^{0} \oplus W^{1}$ and $\mathcal{A}=\{0,1\}$. Let $\mathcal{V}_{00}^{0}$ be the one-dimensional space spanned by the vertex operator defining the vertex operator algebra $W^{0}, \mathcal{V}_{01}^{1}$ the one-dimensional space spanned by the vertex operator defining the $W^{0}$ module $W^{1}, \mathcal{V}_{10}^{1}$ the one-dimensional space spanned by the intertwining operator obtained from the vertex operator defining the $W^{0}$-module $W^{1}$ using skew-symmetry, and let $\mathcal{V}_{00}^{1}, \mathcal{V}_{01}^{0}, \mathcal{V}_{10}^{0}, \mathcal{V}_{11}^{0}$ and $\mathcal{V}_{11}^{1}$ be 0 . Let $\mathbf{1}$ and $\omega$ be the vacuum and Virasoro element of $W^{0}$. Then it is easy to verify that $\left(W, \mathcal{A},\left\{\mathcal{V}_{a_{1} a_{2}}^{a_{3}}\right\}, \mathbf{1}, \omega\right)$ is an intertwining operator algebra. Since $W^{0}$ satisfies the conditions in Theorem 2.5, $W$ is completely extendable. Now choose 
$W^{0}$ and $W^{1}$ such that $\left(W^{0}\right)^{\prime}$ and $\left(W^{1}\right)^{\prime}$ are isomorphic to $W^{0}$ and $W^{1}$, respectively, as $W^{0}$-modules. Then the space of intertwining operators of type $\left(\begin{array}{c}\left(W^{0}\right)^{\prime} \\ W^{1}\left(W^{1}\right)^{\prime}\end{array}\right)$ is isomorphic to the space of intertwining operators of type $\left(\begin{array}{c}W^{0} \\ W^{1} W^{1}\end{array}\right)$. But we know that there is a nonzero intertwining operator of type $\left(\begin{array}{c}\left(W^{0}\right)^{\prime} \\ W^{1}\left(W^{1}\right)^{\prime}\end{array}\right)$ contragredient to the intertwining operator spanning the space $\mathcal{V}_{10}^{1}$ (see [HL2]). Thus the space of all intertwining operators of type $\left(\begin{array}{c}\left(W^{0}\right)^{\prime} \\ W^{1}\left(W^{1}\right)^{\prime}\end{array}\right)$ is nonzero, and consequently the space of intertwining operators of type $\left(\begin{array}{c}W^{0} \\ W^{1} W^{1}\end{array}\right)$ is nonzero. So we see that for the intertwining operator algebra $W, \mathcal{V}_{11}^{0}=0$ is not equal to the space of all intertwining operators of type $\left(\begin{array}{c}W^{0} \\ W^{1} W^{1}\end{array}\right)$. By definition, $W$ is degenerate.

\section{Codes, lattices and completely-extendable intertwining operator algebras}

In this section, for the convenience of the reader, we first recall definitions and properties of linear binary codes and lattices. Then we give the correspondence among codes, lattices and nondegenerate conformal intertwining algebras using a table. A large part of the correspondence was known to or was conjectured by Frenkel, Lepowsky, Meurman and Goddard. The main new thing in this section is that the correspondence given here is mathematically precise because of the precise conformal-field-theoretic analogues of codes and lattices and the results obtained in the preceding sections.

A (binary linear) code of length $n \in \mathbb{N}$ is a subspace of the vector space $\mathbb{Z}_{2}^{n}$ over $\mathbb{Z}_{2}$. The dimension of the subspace is called the dimension of the code. Elements of a code are called codewords. Any element $S$ of $\mathbb{Z}_{2}^{n}$ is a linear combination of the basis elements $(1,0, \ldots, 0), \ldots,(0, \ldots, 0,1)$. The number of nonzero coefficients in the linear combination is called the weight of $S$ and is denoted by $|S|$. A code $\mathcal{S}$ is said to be even if $|S| \in 2 \mathbb{Z}$ for all $S \in \mathcal{S}$ and doubly even if $|S| \in 4 \mathbb{Z}$ for all $S \in \mathcal{S}$. The usual dot product on $\mathbb{Z}_{2}^{n}$ gives a natural nondegenerate symmetric bilinear form on $\mathbb{Z}_{2}^{n}$. The orthogonal space of a code $\mathcal{S}$ in $\mathbb{Z}_{2}^{n}$ with respect to this bilinear form is again a code. It is called the dual code of $\mathcal{S}$ and is denoted $\mathcal{S}^{\circ}$. A code is called self-dual if it is equal to its dual code. For any code $\mathcal{S}$, we have a polynomial

$$
W_{\mathcal{S}}(q)=\sum_{S \in \mathcal{S}} q^{\mathrm{wt}} S
$$


A (rational) lattice of rank $n \in \mathbb{N}$ is a rank $n$ free abelian group $L$ equipped with a rational-valued symmetric $\mathbb{Z}$-bilinear form $(\cdot, \cdot)$. A lattice is nondegenerate if its form is nondegenerate. Let $L$ be a lattice. For $m \in \mathbb{Q}$, we set $L_{m}=\{\alpha \in L \mid(\alpha, \alpha)=m\}$. The lattice $L$ is said to be even if $L_{m}=0$ for any $m \in \mathbb{Q}$ which is not an even integer. A lattice $L$ is said to be integral if the form is integral valued and to be positive definite if the form is positive definite. Even lattices are integral. Let $L_{\mathbb{Q}}=L \otimes_{\mathbb{Z}} \mathbb{Q}$. Then $L_{\mathbb{Q}}$ is an $n$-dimensional vector space over $\mathbb{Q}$ in which $L$ is embedded and the form on $L$ is extended to a symmetric $\mathbb{Q}$-bilinear form on $L_{\mathbb{Q}}$, still denoted by $(\cdot, \cdot)$. The lattice is nondegenerate if and only if this form on $L_{\mathbb{Q}}$ is nondegenerate. The dual of $L$ is the set $L^{\circ}=\left\{\alpha \in L_{\mathbb{Q}} \mid(\alpha, L) \subset \mathbb{Z}\right\}$. This set is a lattice if and only if $L$ is nondegenerate, and in this case, $L^{\circ}$ has as a basis the dual basis of a given basis. The lattice $L$ is said to be self-dual if $L=L^{\circ}$. This is equivalent to $L$ being integral and unimodular, which means that $\left|\operatorname{det}\left(\left(\alpha_{i}, \alpha_{j}\right)\right)\right|=1$. For any lattice $L$, we have the associated theta series

$$
\Theta_{L}(q)=\sum_{\alpha \in L} q^{\frac{1}{2}(\alpha, \alpha)}
$$

Given a completely-extendable conformal intertwining algebra $W$ such that $\operatorname{dim} W_{(n)}<\infty$ for $n \in \mathbb{C}$, let

$$
\chi_{W}(q)=q^{-\frac{c}{24}} \sum_{n \in \mathbb{C}}\left(\operatorname{dim} W_{(n)}\right) q^{n} .
$$

It is called the character or graded dimension or the partition function of $W$.

Now see the table for the precise correspondence among codes, lattices and completely-extendable conformal intertwining algebras. This table fills some blanks in a table in $\mathrm{G}$ and gives more items than the one in $\mathrm{G}$. There are constructions of lattices from codes and abelian intertwining algebras from lattices (see [CN] and DL2]).

In fact this correspondence can be formulated more conceptually in terms of the language of categories and related results can be established. Some of the constructions mentioned above should be viewed as canonical functors and others should be viewed as compositions of these canonical functors with some twist functors in the categories of lattices and completely-extendable conformal intertwining algebras. We shall discuss this formulation and these results in a future paper. 


\begin{tabular}{|l|l|l|}
\hline Codes & Lattices & $\begin{array}{l}\text { Completely-extendable confor- } \\
\text { mal intertwining algebras }\end{array}$ \\
\hline Even codes & Integral lattices & $\begin{array}{l}\text { Meromorphic completely- } \\
\text { extendable conformal intertwin- } \\
\text { ing algebras }\end{array}$ \\
\hline $\begin{array}{l}\text { Doubly } \\
\text { even codes }\end{array}$ & Even lattices & $\begin{array}{l}\text {ZZ-graded completely-extendable } \\
\text { conformal intertwining algebras }\end{array}$ \\
\hline & Positive-definite lattices & $\begin{array}{l}\text { Completely-extendable inter- } \\
\text { twining operator algebras }\end{array}$ \\
\hline & $\begin{array}{l}\text { Positive definite and in- } \\
\text { tegral lattices }\end{array}$ & $\begin{array}{l}\text { Meromorphic completely- } \\
\text { extendable intertwining operator } \\
\text { algebras }\end{array}$ \\
\hline Golay code & Leech lattice & $\begin{array}{l}\mathbb{Z} \text {-graded completely-extendable } \\
\text { intertwining operator algebras }\end{array}$ \\
\hline Lengths & Ranks & $\begin{array}{l}\text { Moonshine module vertex oper- } \\
\text { ator algebra }\end{array}$ \\
\hline Weights & Square lengths & Central charges (or ranks) \\
\hline Dual codes & Dual lattices & $\begin{array}{l}\text { Weights } \\
\text { conformal of completely-extendable }\end{array}$ \\
\hline $\begin{array}{l}\text { Self-dual } \\
\text { codes }\end{array}$ & Self-dual lattices & $\begin{array}{l}\text { Self-dual completely-extendable } \\
\text { conformal intertwining algebras }\end{array}$ \\
\hline & Nondegenerate lattices & $\begin{array}{l}\text { Nondegenerate completely- } \\
\text { extendable conformal intertwin- } \\
\text { ing algebras }\end{array}$ \\
\hline $\mathbb{F}_{2}^{n}$ & $L_{\mathbb{Q}}$ & Complete extensions \\
\hline$W_{\mathcal{S}}(q)$ & $\Theta_{L}(q)$ & $\chi_{W}(q)$ \\
\hline & & \\
\hline
\end{tabular}




\section{References}

[B] R. E. Borcherds, Vertex algebras, Kac-Moody algebras, and the Monster, Proc. Natl. Acad. Sci. USA 83 (1986), 3068-3071.

[C] J. H. Conway, A characterisation of Leech's lattice, Invent. Math. 7 (1969), 137-142.

[CN] J. H. Conway, N. J. Sloane, Sphere packings, lattices and groups, 3rd edition, Grundlehren der Mathematischen Wissenschaften, Vol. 290, Springer-Verlag, New York, 1999.

[DGM] L. Dolan, P. Goddard and P. Montague, Conformal field theory of twisted vertex operators, Nucl. Phys. B338 (1990), 529-601.

[DL1] C. Dong and J. Lepowsky, Abelian intertwining algebras - a generalization of vertex operator algebras, in: Proc. AMS Summer Research Institute on Algebraic Groups and Their Generalizations, Pennsylvania State University, 1991, ed. W. J. Haboush and B. J. Parshall, Proc. Symp. Pure Math., Vol 56, Part 2, American Mathematical Society, Providence, 1994, 261-293.

[DL2] C. Dong and J. Lepowsky, Generalized Vertex Algebras and Relative Vertex Operators, Progress in Math., Vol. 112, Birkhäuser, Boston, 1993.

[FFR $]$ A. J. Feingold, I. B. Frenkel, J. F. X. Ries, Spinor construction of vertex operator algebras, triality and $E_{8}^{(1)}$, Contemporary Math., Vol. 121, Amer. Math. Soc., Providence, 1991.

[FHL] I. B. Frenkel, Y.-Z. Huang and J. Lepowsky, On axiomatic approaches to vertex operator algebras and modules, preprint, 1989; Memoirs Amer. Math. Soc. 104, 1993.

[FLM1] I. B. Frenkel, J. Lepowsky and A. Meurman, A natural representation of the Fischer-Griess Monster with the modular function $J$ as character, Proc. Natl. Acad. Sci. USA 81 (1984), 3256-3260.

[FLM2] I. B. Frenkel, J. Lepowsky, and A. Meurman, Vertex operator algebras and the Monster, Pure and Appl. Math., Vol. 134, Academic Press, New York, 1988. 
[G] P. Goddard, Meromorphic conformal field theory, in: Infinitedimensional Lie algebras and groups (Luminy-Marseille, 1988), ed. V. Kac, Adv. Ser. Math. Phys., Vol. 7, World Sci. Publishing, Teaneck, 1989, 556-587.

[H1] Y.-Z. Huang, Geometric interpretation of vertex operator algebras, Proc. Natl. Acad. Sci. USA 88 (1991), 9964-9968.

[H2] Y.-Z. Huang, A theory of tensor products for module categories for a vertex operator algebra, IV, J. Pure Appl. Alg. 100 (1995), 173-216.

[H3] Y.-Z. Huang, A nonmeromorphic extension of the moonshine module vertex operator algebra, in: Moonshine, the Monster and related topics, Proc. Joint Summer Research Conference, Mount Holyoke, 1994, ed. C. Dong and G. Mason, Contemporary Math., Vol. 193, Amer. Math. Soc., Providence, 1996, 123-148.

[H4] Y.-Z. Huang, Virasoro vertex operator algebras, (nonmeromorphic) operator product expansion and the tensor product theory, J. Alg. 182 (1996), 201-234.

[H5] Y.-Z. Huang, Intertwining operator algebras, genus-zero modular functors and genus-zero conformal field theories, in: Operads: Proceedings of Renaissance Conferences, ed. J.-L. Loday, J. Stasheff, and A. A. Voronov, Contemporary Math., Vol. 202, Amer. Math. Soc., Providence, 1997, 335-355.

[H6] Y.-Z. Huang, Two-dimensional conformal geometry and vertex operator algebras, Progress in Mathematics, Vol. 148, 1997, Birkhäuser, Boston.

[H7] Y.-Z. Huang, Genus-zero modular functors and intertwining operator algebras, Internat. J. Math. 9 (1998), 845-863.

[H8] Y.-Z. Huang, Generalized rationality and a "Jacobi identity" for intertwining operator algebras, Selecta Mathematica, New Series, to appear.

[H9] Y.-Z. Huang, Intertwining algebras, in preparation. 
[HL1] Y.-Z. Huang and J. Lepowsky, A theory of tensor products for module categories for a vertex operator algebra, I, Selecta Mathematica, New Series 1 (1995), 699-756.

[HL2] Y.-Z. Huang and J. Lepowsky, A theory of tensor products for module categories for a vertex operator algebra, II, Selecta Mathematica, New Series 1 (1995), 757-786.

[HL3] Y.-Z. Huang and J. Lepowsky, Tensor products of modules for a vertex operator algebras and vertex tensor categories, in: Lie Theory and Geometry, in honor of Bertram Kostant, ed. R. Brylinski, J.-L. Brylinski, V. Guillemin, V. Kac, Birkhäuser, Boston, 1994, 349-383.

[HL4] Y.-Z. Huang and J. Lepowsky, A theory of tensor products for module categories for a vertex operator algebra, III, J. Pure Appl. Alg. 100 (1995), 141-171.

[HL6] Y.-Z. Huang and J. Lepowsky, Intertwining operator algebras and vertex tensor categories for affine Lie algebras, Duke Math. J. 99 (1999), 113-134.

[HM1] Y.-Z. Huang and A. Milas, Intertwining operator superalgebras and vertex tensor categories for superconformal algebras, I, to appear.

[HM2] Y.-Z. Huang and A. Milas, Intertwining operator superalgebras and vertex tensor categories for superconformal algebras, II, Trans. Amer. Math. Soc., to appear.

Department of Mathematics, Rutgers University, 110 FrelinghuySEn RD., Piscataway, NJ 08854-8019

E-mail address: yzhuang@math.rutgers.edu 